\title{
Regularity at the free boundary of two-dimensional stationary harmonic maps
}

\author{
by \\ Michael GRÜTER \\ Fachbereich Mathematik Universität des Saarlandes Im Stadtwald D-66123 Saarbrïcken \\ grueter@math.uni-sb.de
}

ABSTRACT. - It is shown that the regularity problem at the free boundary of two-dimensional stationary harmonic maps can be reduced to the corresponding problem for two-dimensional minimal surfaces and that the analogous regularity results hold true.

(c) 1998 L'Association Publications de l'Institut Henri Poincaré. Published by Elsevier B.V. All rights reserved

RÉSUMÉ. - On montre que le problème de la régularité à la frontière libre d'une application harmonique stationnaire bidimensionnelle peut être réduit au problème analogue pour les surfaces minimales, et que les résultats de régularité correspondants sont encore vrais.

(C) 1998 L'Association Publications de l'Institut Henri Poincaré. Published by Elsevier B.V. All rights reserved

\section{INTRODUCTION}

Since the direct method of the calculus of variations in general produces weak solutions one of the basic questions is the regularity problem. Is the generalized solution a classical solution, does it have singularities, how large can the singular set be? In the theory of harmonic mappings between Riemannian manifolds there is a big difference - regarding these questions - between the two-dimensional and the higher dimensional case. If the domain of definition of the mappings has dimension $n \geq 3$ even

1991 Mathematics Subject Classification: 49 N 60, 35 R 35, 49 Q 05. 
minimizers of the energy functional may have singular points. A simple example is the mapping $u_{0}: \mathbb{R}^{n} \rightarrow S^{n-1}$ given by $u_{0}(x)=x /|x|$, c.f. [17].

Here, we are concerned with the two-dimensional case. In contrast to higher dimensions it had already been proved by C.B. Morrey in [18] that in two dimensions energy minimizing harmonic maps are regular. In fact, Morrey's method works more generally for variational integrals growing quadratically w.r.t. the gradient of the map. However, in many situations one is interested not only in the minimizers but also wants to know the whole set of critical points.

As is well known there is an intimate connection between twodimensional harmonic maps and two-dimensional minimal surfaces. For example, if $N$ is a Riemannian manifold it turns out that every harmonic map $u: S^{2} \rightarrow N$ is in fact a parametric minimal surface (or constant). In general, a harmonic map is a parametric minimal surface (possibly with branch points) provided it is conformally parametrized. In this case the energy integral equals twice the area of the mapping.

Since in [6] the author proved that weak $\mathrm{H}$-surfaces with finite area are regular in the interior it follows that conformally parametrized harmonic maps having finite energy also are regular in the interior. In [10], see also [3], it was then shown that the method of [6] can be modified to work for minimal surfaces with a free boundary. Later, technical improvements and generalizations were given in [4], [11], [9], [14], and [15]. The behaviour of minimizers near the free boundary had before been investigated by $\mathrm{H}$. Lewy [16] and W. Jäger [13]. For more references the reader is referred to the relevant sections of [1] and [2] as well as to the earlier monographs by J.C.C. Nitsche [19], and [20].

Let us now turn to harmonic maps which are not necessarily conformally parametrized. Nevertheless, in the smooth case there still is a certain holomorphic function (a holomorphic quadratic differential if the domain of definition is a Riemann surface) associated to the map. For example, if $u \in C^{2}\left(\mathbb{R}^{2}, \mathbb{R}^{N}\right)$ satisfies

$$
\Delta u^{k}=0 \quad \text { in } \mathbb{R}^{2} \quad(k=1, \ldots N) .
$$

then the function $\Phi: \mathbb{C} \rightarrow \mathbb{C}(z=x+i y)$ given by

$$
\Phi(z):=\left|u_{x}\right|^{2}-\left|u_{y}\right|^{2}-2 i u_{x} \cdot u_{y}
$$

can easily be checked to be holomorphic.

In the case that $u$ is only known to be weakly harmonic one may argue as follows. Suppose that in addition $u$ is a stationary point of the energy 
integral w.r.t. inner variations (see section 2 for precise definitions). Then, by well known arguments it can be shown that the function $\Phi$ defined in (2) (respectively its analogue in the Riemannian case) is a weakly holomorphic $L^{1}$-function. Using this observation and a simple construction c.f. [8], and [22] for a slightly more complicated argument, the question of interior regularity for stationary harmonic maps could be reduced to the case treated in [6].

In this paper we are going to show how these arguments can be modified to give regularity for stationary harmonic maps with a frec boundary.

Let us mention here that the regularity question for weakly harmonic maps in the interior case has successfully been solved by F. Hélein in [12]. Later, J. Qing [21] using Hélein's result was able to show regularity at the fixed boundary (Dirichlet conditions) for weakly harmonic maps. The case of a free boundary is currently under investigation.

This paper is organized as follows. In section 2 we treat the Euclidean case in fair detail hecause already here the main idea hecomes clear and one can avoid the technical complications necessary in other cases. Section 3 is then devoted to more general problems that can be handled in this way. In particular, we indicate how to treat more general functionals, the Riemannian case, as well as the case where the supporting surface is allowed to have a non-empty boundary (a kind of Signorini problem).

\section{THE MODEL CASE}

Here, we consider the following situation.

The two-dimensional Euclidean space $\mathbb{R}^{2}$ is identified with the complex plane $\mathbb{C}$, and accordingly points in $\mathbb{R}^{2}$ are written as $w=(u, v)=u+i v$.

As a parameter domain we choose the open semi-disk

$$
B^{+}:=\left\{w \in \mathbb{R}^{2}:|w|<1, v>0\right\} .
$$

Let $C$ denote the closed circular arc

$$
C:=\left\{w \in \mathbb{R}^{2}:|w|=1, v \geq 0\right\},
$$

and $I$ the open interval

$$
I:=\left\{u \in \mathbb{R}^{2}:|w|<1, v=0\right\}=(-1,1) \times\{0\},
$$

so that $\partial B^{+}=C \dot{\cup} I$ (disjoint union).

Vol. 15, n 2-1998. 
Furthermore, assume that $S$ is a smooth two-dimensional surface in $\mathbb{R}^{3}$ - the so called supporting surface. We want to investigate the regularity properties of harmonic maps

$$
X: B^{+} \rightarrow \mathbb{R}^{3}
$$

which map $I$ into $S$, i.e. the boundary values $X_{I}:=X_{\mid I}$ are allowed to vary freely on the supporting surface. Of course, the only problem is the regularity near the free boundary $I$. It is instructive to look at the following

Example. - On $B^{+}$consider the real-valued function $f \in H_{2}^{1}\left(B^{+}\right)$ defined by

$$
f(w):=\log \left(\log \left(\frac{e}{|w|}\right)\right)
$$

so that $f_{\mid C} \equiv 0$.

Denote by $z_{0} \in H_{2}^{1}\left(B^{+}\right)$the harmonic function having the same boundary values as $f$, e.g. minimize the Dirichlet integral

$$
D(z):=\int_{B^{+}}|D z|^{2}
$$

in the class

$$
\mathcal{C}:=\left\{z \in H_{2}^{1}\left(B^{+}\right): z-f \in \stackrel{0}{H}_{2}^{1}\left(B^{+}\right)\right\} .
$$

As a supporting surface we take the hyperplane

$$
S:=\left\{\left(x^{1}, x^{2}, x^{3}\right) \in \mathbb{R}^{3}: x^{2}=0\right\}
$$

and define $X \in H_{2}^{1}\left(B^{+}, \mathbb{R}^{3}\right)$ by

$$
X(u, v):=\left(u, v, z_{0}(u, v)\right) .
$$

Obviously, we have

$$
\begin{aligned}
& \Delta X^{k}=0 \quad \text { in } B^{+} \quad(k=1,2,3), \\
& X_{\mid C}=I d_{\mid C}, \\
& X(I) \subset S
\end{aligned}
$$

but $X \notin C^{0}\left(\overline{B^{+}}\right)$; indeed, $X$ is not even bounded near the free boundary. 
Remarks. - Of course, one can construct discontinuous bounded harmonic maps in the same way.

Note that the finiteness of the Dirichlet integral or the boundedness of a harmonic function imply that singular sets of capacity zero in the interior are removable.

In view of this counterexample we are led to consider stationary harmonic maps. To be more precise we make the following assumptions.

Let

$$
\mathcal{C}(S):=\left\{X \in H_{2}^{1}\left(B^{+}, \mathbb{B}^{3}\right): X(I) \subset S\right\} .
$$

where the free boundary condition $X(I) \subset S$ is to be understood in the sense that the $L^{2}$-trace $X_{I}$ of $X$ maps almost all of $I$ into $S$.

Here, the two-dimensional surface $S \subset \mathbb{R}^{3}$ is supposed to satisfy the following

\section{Assumption (A)}

There are numbers $\rho_{0}>0, K_{0} \geq 0$ and $K \geq 1$ such that the following holds:

For each point $x_{0} \in S$ there is a (full) neighbourhood $\mathcal{U}$ of $x_{0}$ in $\mathbb{R}^{3}$ and a $C^{2}$-diffeomorphism $x=h(y)$ of $\mathbb{R}^{3}$ onto itself with the following two properties. Firstly, the inverse $h^{-1}$ maps $x_{0}$ onto 0 and $\mathcal{U}$ onto the open ball $B_{\rho_{0}}=\left\{y \in \mathbb{R}^{3}:|y|<\rho_{0}\right\}$ such that $S \cap \mathcal{U}$ corresponds to the set $\left\{y \in B_{\rho_{0}}: y^{3}=0\right\}$ on the hyperplane $\left\{y^{3}=0\right\}$.

Secondly, if we set ${ }^{1}$

$$
g_{j k}(y):=h_{y^{j}}(y) \cdot h_{y^{k}}(y)
$$

then

$$
K^{-1}|\xi|^{2} \leq g_{j k}(y) \xi^{j} \xi^{k} \leq K|\xi|^{2}
$$

for all $\xi, y \in \mathbb{R}^{3}$, as well as

$$
\left|\frac{\partial g_{j k}}{\partial y^{l}}\right|(y) \leq K_{0}
$$

for all $y \in \mathbb{R}^{3}$ and $j, k, l \in\{1,2,3\}$.

Obviously, every compact (without boundary) $C^{2}$-submanifold of $\mathbb{R}^{3}$ satisfies assumption (A). Each submanifold of $\mathbb{R}^{3}$, compact or non-compact, for which assumption (A) holds, is a complete Riemannian manifold with respect to the induced metric of $\mathbb{R}^{3}$. For non-compact surfaces $S$, assumption (A) imposes a certain uniformity condition on the metric

\footnotetext{
I Here and in the following the summation convention is used.
} 
$d s^{2}=g_{j k}(y) d y^{j} d y^{k}$ at infinity and is thus somewhat more stringent than the sole condition $S \in C^{2}$. Therefore, a $C^{2}$-submanifold of $\mathbb{R}^{3}$ satisfying (A) will be called a strict $C^{2}$-surface in $\mathbb{R}^{3}$.

DEFINITION 1. - An admissible variation of a surface $X \in \mathcal{C}(S)$ is a family of surfaces $X_{t} \in \mathcal{C}(S),|t|<t_{0}$ for some number $t_{0}>0$, where $\left\{X_{t}\right\}_{|t|<t_{0}}$ is of one of the following two types.

TYPE I. - (Inner variations)

The surfaces $X_{l}$ are of the form $X_{l}=X \circ \tau_{l}$, where $\left\{\tau_{l}\right\}_{|l|<l_{l}}$ is a family of diffeomorphisms from $\overline{B^{+}}$to itself such that $\tau_{0}$ is the identity, $\tau(w, t)=\tau_{t}(w)$ is of class $C^{1}$ on $\overline{B^{+}} \times\left(-t_{0}, t_{0}\right)$, and such that

$$
\operatorname{spt} \frac{\partial \tau}{\partial t} \subset B^{+} \cup I
$$

TYPE II. - (Outer variations)

The surfaces $X_{+}$are of the form

$$
X_{t}(w)=X(w)+t \Psi(w, t)
$$

where

$$
D(\Psi(\cdot, t)) \leq C \quad \text { for }|t|<t_{0}
$$

with $C$ independent of $t$, spt $\Psi(\cdot, t) \subset K \subset B^{+}(\lrcorner I$, with $K$ compact and independent of $t$, and such that

$$
\Psi(w . t) \rightarrow \Psi^{0}(w) \text { as } t \rightarrow 0
$$

for a.e. $w \in B^{+}$for some $\Psi^{0} \in H_{2}^{1}\left(B^{+}, \mathbb{R}^{3}\right)$.

Remarks. - Note, that condition ( 1 ) is equivalent to the requirement that the diffeomorphisms $\tau_{t}$ leave a neighbourhood of $C$ fixed. For an admissible variation of type II it follows from (3) that for $\mathcal{H}^{1}$-almost all $w \in I$ the vector $\Psi^{0}(w)$ is tangent to $S$ at $X(w)$.

Definition 2. - We call $X \in \mathcal{C}(S)$ a stationary harmonic map if the Dirichlet integral $D(X)$ is stationary w.r.t. every admissible variation $\left\{X_{t}\right\}$ of $X$, i.e. if

$$
\frac{d}{d t} D\left(X_{t}\right)_{\mid t=0}=0
$$

CONSEquences OF STATIONNARITY. - As is well known, stationarity w.r.t. admissible variations of type I implies

$$
\int_{B^{+}}\left[\left(\left|X_{u}\right|^{2}-\left|X_{v}\right|^{2}\right)\left(\xi_{u}^{1}-\xi_{v}^{2}\right)+2 X_{u} \cdot X_{v}\left(\xi_{v}^{1}+\xi_{u}^{2}\right)\right] d u d v=0
$$


for any vectorfield $\xi \in C_{c}^{1}\left(B^{+} \cup I, \mathbb{R}^{2}\right)$ which is "real" on $I$ (i.e. tangential along $I$ ).

On the other hand, from admissible variations of type II we conclude

$$
\int_{B^{+}}\left[X_{u} \cdot \Psi_{u}^{0}+X_{v} \cdot \Psi_{v}^{0}\right] d u d v=0
$$

for any $\Psi^{0} \in H_{2}^{1}\left(B^{+}, \mathbb{R}^{3}\right)$ such that $\Psi_{\mid C}^{0} \equiv 0$ and $\Psi^{0}(w) \in \operatorname{Tan}_{\mathrm{X}(w)} S$ for $\mathcal{H}^{1}$-almost all $w \in I$.

Taking $\xi \in C_{c}^{1}\left(B^{+}, \mathbb{R}^{2}\right)$ in (5) one easily checks that

$$
\Phi(w):=\left(\left|X_{u}(w)\right|^{2}-\left|X_{v}(w)\right|^{2}\right)-2 i X_{u}(w) \cdot X_{u}(w)
$$

is a holomorphic function on $B^{+}$.

Alternatively, this can also be deduced from the fact that (6) implies (take $\Phi^{0} \in \stackrel{0}{H}_{2}^{1}\left(B^{+}, \mathbb{R}^{3}\right)$ ) the harmonicity of $X$ :

$$
\Delta X^{k}=0 \quad \text { in } B^{+} \quad(k=1,2,3) .
$$

An important step in the regularity proof will be the fact that (5) implies that $\Phi$ is regular up to the boundary $I$. Although this is more or less well known, for the convenience of the reader we repeat the short proof given in [23], see also [5]. First, note that (5) is equivalent to the equation

$$
\int_{B^{+}} \operatorname{Re}(\Phi \bar{\partial} \xi) d u d v=0
$$

for every $\xi \in C_{c}^{1}(B, \mathbb{C})$ with $\xi_{I}$ real.

Here, $B:=\{w \in \mathbb{C}:|w|<1\}$ is the unit disk, and $\bar{\partial}=\frac{1}{2}\left(\partial_{u}+i \partial_{w}\right)$, $\partial=\frac{1}{2}\left(\partial_{u}-i \partial_{v}\right)$ denote the Wirtinger operators. Now, (9) implies that in a weak sense $-\Phi$ is real-valued on $I$. Thus, if $\Phi$ is extended to $B$ as an $L^{2}$-function by

$$
\Phi(w):=\Phi^{*}(w):=\overline{\Phi(\bar{w})} \text { for } w \in B \text { with } \operatorname{Im}(w)<0 .
$$

it follows - by the famous Schwarz reflection principle - that $\Phi$ is (weakly) holomorphic on $B$. To see this consider any $\xi \in C_{c}^{1}(B, \mathbb{C})$ and using (9) and $\bar{\partial}\left(\xi^{*}\right)=(\bar{\partial} \xi)^{*}$ check that

$$
\int_{B} \operatorname{Re}(\Phi \bar{\partial} \xi) d u d v=\int_{B^{+}} \operatorname{Re}\left(\Phi \bar{\partial}\left(\xi+\xi^{*}\right)\right) d u d v=0 .
$$


because $\xi+\xi^{*} \in C_{c}^{1}\left(B^{+} \cup I, \mathbb{C}\right)$ and $\left(\xi+\xi^{*}\right)_{\mid I}=2 \operatorname{Re} \xi_{\mid I}$. Thus, the extended function $\Phi$ is $L^{2}$-equivalent to a holomorphic function on $B$ (again called $\Phi$ ). In particular, we conclude from (10) that

$\Phi$ is real-valued on $I$,

which can be interpreted as

$$
X_{u} \cdot X_{v}=0 \text { on } I
$$

Remarks. - As has first been observed by W. Jäger [13] relation (6) implies that in a weak sense the surface $X$ is orthogonal to the supporting surface $S$ along $I$. Hence, for smooth (up to the free boundary $I$ ) maps $X \in \mathcal{C}(S)$ which are stationary w.r.t. outer variations the equation (12) is automatically satisfied in view of the fact that in this case $X_{u t}$ is tangent to $S$ along $I$.

Now, define $\tilde{\Phi}: B \rightarrow \mathbb{C}$ by

$$
\tilde{\Phi}(w):=-\frac{1}{4} \int_{0}^{w} \Phi(\tilde{w}) d \tilde{w}
$$

so that

$$
\partial \dot{\Phi}=-\frac{1}{4} \Phi \quad \text { on } B
$$

and from (11) we conclude

$$
\tilde{\Phi} \text { is real-valued on } I \text {. }
$$

The holomorphicity of $\tilde{\Phi}$ obviously implies that

$$
\tilde{\Phi} \in H_{2, \text { loc }}^{1}(B, \mathbb{C}) \text {. }
$$

Suppose now that $X \in \mathcal{C}(S)$ is a stationary harmonic map in the sense of Definition 2 and define

$$
Y: B^{+} \rightarrow \mathbb{R}^{5}=\mathbb{R}^{3} \times \mathbb{R}^{2}=\mathbb{R}^{3} \times \mathbb{C}
$$

by

$$
Y(w)=(X(w), \bar{w}+\tilde{\Phi}(w)) .
$$

An easy calculation, c.f. [8], shows that $Y$ now is conformally parametrized on $B^{+}$, i.e.

$$
\left|Y_{u}\right|^{2}=\left|Y_{v}\right|^{2} \quad \text { and } \quad Y_{u} \cdot Y_{v}=0 .
$$


Since in addition

$$
\Delta Y^{k}=0 \quad \text { in } B^{+} \quad(k=1,2, \ldots, 5)
$$

we conclude that $Y$ is a two-dimensional parametric minimal surface in $\mathbb{R}^{5}$.

The next step is to show that $Y$ is a stationary minimal surface for some new free boundary problem. To this end let

$$
\tilde{S}:=S \times(\mathbb{R} \times\{0\}) \subset \mathbb{R}^{5}
$$

so that $\tilde{S}$ is a three-dimensional surface in $\mathbb{R}^{5}$, namely the right cylinder over $S$ in $\mathbb{R}^{4}$ viewed as a subset of $\mathbb{R}^{5}$. As is easily checked, the $C^{2}$ submanifold $\tilde{S}$ of $\mathbb{R}^{5}$ is in fact a strict $C^{2}$-submanifold of $\mathbb{R}^{5}$ in the (appropriately generalized) sense of assumption (A).

For any $0<R<1$ the new class of comparison surfaces is defined by

$$
\mathcal{C}_{R}(\tilde{S}):=\left\{\tilde{X} \in H_{2}^{1}\left(B_{R}^{+}, \mathbb{R}^{5}\right): \tilde{X}\left(I_{R}\right) \subset \tilde{S}\right\}
$$

Since for a.e. $w \in I$ we have $X(w) \in S$ and $\bar{w}+\tilde{\Phi}(w) \in \mathbb{R}$ it follows that $Y \in \mathcal{C}_{R}(\tilde{S})$.

Lemma. - For any $0<R<1$ the map $Y \in \mathcal{C}_{R}(\tilde{S})$ defined above is a stationary point of the Dirichlet integral (w.r.t. inner and outer variations).

Remarks. - Since $Y$ is conformally parametrized the analogue (5') of equation (5) is obviously satisfied, in fact (5') will hold for any $\xi \in C^{1}\left(\overline{B_{R}^{+}}, \mathbb{R}^{2}\right)$. However, for the regularity proof no inner variations will be needed once it is known that the solution is conformally parametrized.

Proof. - Because of the preceding remark it suffices to check the analogue of (6), i.e.

$$
\int_{B_{R}^{+}}\left[Y_{u} \cdot \Psi_{u}+Y_{v} \cdot \Psi_{v}\right] d u d v=0
$$

holds for any $\Psi \in H_{2}^{1}\left(B_{R}^{+}, \mathbb{R}^{5}\right)$ such that $\Psi_{\mid C_{R}} \equiv 0$ and $\Psi(w) \in \operatorname{Tan}_{Y(w)} \tilde{S}$ for $\mathcal{H}^{1}$-almost all $w \in I_{R}$. To see this, first note that for any $y=(x, z) \in \tilde{S}$ we have

$$
\operatorname{Tan}_{y} \tilde{S}=\operatorname{Tan}_{x} S \times(\mathbb{R} \times\{0\}) .
$$

Next, write any $\Psi$ as in $\left(6^{\prime}\right)$ as

$$
\Psi=\left(\Psi^{0}, \psi\right)
$$

Vol. $15, n^{\circ} 2-1998$. 
Then, in view of (6) the conditions on $\Psi$ imply that

$$
\begin{aligned}
& \int_{B_{R}^{+}}\left[Y_{u} \cdot \Psi_{u}+Y_{v} \cdot \Psi_{v}\right] d u d v= \\
& \int_{B_{R}^{+}}\left((1,0)+\tilde{\Phi}_{u}\right) \cdot \psi_{u}+\left((0,-1)+\tilde{\Phi}_{v}\right) \cdot \psi_{v} d u d v= \\
& -\int_{I_{R}}\left((0,-1)+\tilde{\Phi}_{v}\right) \cdot \psi d u=-\int_{I_{R}}\left(0,-1-\frac{1}{4}\left(\left|X_{u}\right|^{2}-\left|X_{v}\right|^{2}\right)\right) \cdot \psi d u=0 .
\end{aligned}
$$

Here, we used (14) together with (11) and the fact that

$$
\tilde{\Phi}_{v}=-\frac{1}{4} i \Phi
$$

Thus, (6') has been established and the Lemma is proved.

In this way we have reduced the regularity problem for stationary harmonic maps to the analogous regularity problem for stationary minimal surfaces. Therefore, the reasoning of [10] can be applied almost verbatim to deduce the continuity of $Y$ up to the free boundary $I_{R}$. This in particular implies the continuity of our original harmonic map $X$.

THEOREM 1. - Let $S \subset \mathbb{R}^{3}$ be a strict $C^{2}$-surface, and suppose that $X(w)$ is a stationary harmonic map in the class $\mathcal{C}(S)$. Then $X(w)$ is continuous on $B^{+} \cup I$.

Remarks. - For a detailed proof of the corresponding result for stationary minimal surfaces the reader is referred to the nice presentation in section 7.6 of [2]. One easily checks that the restriction to two-dimensional surfaces in $\mathbb{R}^{3}$ is not essential.

Higher REgularity. - Once continuity of $X$ on $B^{+} \cup I$ is known one can prove higher regularity by methods well known to experts, e.g. Höldercontinuity, $C^{m, \alpha}$-regularity, as well as analyticity, provided the supporting surface is sufficiently regular. In particular, for a strict $C^{2}$-surface as in Theorem 1 one gets $X \in C^{1, \alpha}\left(B^{+} \cup I, \mathbb{R}^{3}\right)$ for any $\alpha \in(0,1)$, and the image surface $X\left(B^{+} \cup I\right)$ meets the supporting surface $S$ orthogonally in the classical sense. For more precise statements compare section 7.8 of [2].

\section{GENERALIZATIONS}

First, we are going to look at more general functionals than the Dirichlet integral. We shall only outline the arguments indicating the necessary changes from the conformally parametrized case. 
In [7] it was shown that every conformally invariant variational integral on $\mathbb{R}^{2}$

$$
I[u]:=\int F(u, D u)
$$

where

$$
F: \mathbb{R}^{N} \times \mathbb{R}^{2 N} \rightarrow \mathbb{R}
$$

satisfies

$$
m m_{1}|p|^{2} \leq F(u, p) \leq m_{2}|p|^{2}
$$

as well as

$$
F(\cdot, p) \in C^{1}\left(\mathbb{R}^{N}\right), F(u, \cdot) \in C^{2}\left(\mathbb{R}^{2 N}\right)
$$

can be written in the form ${ }^{2}$

$$
I[u]=\int G_{j k}(u) D u^{j} \cdot D u^{k}+B_{j k}(u) \operatorname{det}\left(D u^{j}, D u^{k}\right) .
$$

Here, the $(n \times n)$-matrix $\left(G_{j k}\right)_{j, k=1, \ldots, N}$ is symmetric and positive definite, while $\left(B_{j k}\right)_{j, k=1, \ldots, N}$ is skew-symmetric. It follows from [8] that the interior regularity theory for stationary points of (2) can be reduced to the case where the solutions are conformally parametrized. Hence, the results of [6] are applicable.

A prominent example of such a functional is

$$
\int_{\Omega}|D u|^{2}+4 Q(u) \cdot\left(u_{x} \wedge u_{y}\right) d x d y
$$

for a domain $\Omega \subset \mathbb{R}^{2}, u \in H_{2}^{1}\left(\Omega, \mathbb{R}^{3}\right)$, and a smooth vector field $Q$ on $\mathbb{R}^{3}$.

The corresponding Euler equations are

$$
\Delta u=2 \operatorname{div} Q(u) u_{x} \wedge u_{y} \quad \text { in } \Omega
$$

and if $u$ is conformally parametrized it follows that at regular points the mean curvature of the surface $u(\Omega) \subset \mathbb{R}^{3}$ is given by $H(u)=\operatorname{div} Q(u)$.

Let us now return to the general functional (2).

If $u$ is a stationary point of the integral $I$ the function $(z=x+i y \in$ $\mathbb{C}=\mathbb{R}^{2}$ )

(5) $\Phi(z):=G_{j k}(u) \frac{\partial u^{j}}{\partial x} \frac{\partial u^{k}}{\partial x}-G_{j k}(u) \frac{\partial u^{j}}{\partial y} \frac{\partial u^{k}}{\partial y}-2 i G_{j k}(u) \frac{\partial u^{j}}{\partial x} \frac{\partial u^{k}}{\partial y}$

\footnotetext{
${ }^{2}$ Summation convention for $j, k=1,2 \ldots, N$.
} 
is holomorphic in the interior. Here, stationarity is to be understood w.r.t. inner and outer variations (always fixing the boundary values). That $\Phi$ does not depend on the coefficients $\left(B_{j k}\right)$ follows from the fact that the integral

$$
\int B_{j k}(u) \operatorname{det}\left(D u^{j} . D u^{k}\right)
$$

is invariant under orientation preserving diffeomorphisms of the domain of definition of the mapping $u$. Hence, inner variations of this integral do not contribute to the first variation.

Suppose now that $S$ is a k-dimensional $(1 \leq k<N)$ strict $C^{2}$ submanifold of $\mathbb{R}^{N}$ in the (suitably generalized) sense of assumsption (A) in section 2 .

As before, we define the class

$$
\mathcal{C}(S):=\left\{u \in H_{2}^{1}\left(B^{+}, \mathbb{R}^{N}\right): u(I) \subset S\right\} ;
$$

the notion of admissible variation is defined as in Definition 1, and stationary points of the integral $I$ are defined as in Definition 2 . In the conformally parametrized case the integral (3) has been treated in [11].

By the same reasoning as in the model case we may again conclude that

$$
\Phi \text { is real-valued on } I \text {. }
$$

Geometrically speaking this condition means that the tangent vectors $u_{r}$. and $u_{y}$ are orthogonal to each other along the free boundary $I$ if $\mathbb{R}^{N}$ is equipped with the Riemannian metric given by $\left(G_{j k}\right)_{j, k=1, \ldots, N}$.

The original problem is now replaced by a free boundary problem in

$$
\mathcal{M}:=\left(\mathbb{R}^{N},\left(G_{j k}\right)\right) \times\left(\mathbb{R}^{2},\left(\delta_{\alpha, i}\right)\right)
$$

for the $(k+1)$-dimensional supporting submanifold

$$
\tilde{S}:=S \times(\mathbb{R} \times\{0\}) \subset \mathcal{M}
$$

Note, that $\mathcal{M}$ is topologically just $\mathbb{R}^{N+2}$ but with a different Riemannian structure.

If $u_{0} \in \mathcal{C}(S)$ is a stationary point of $I$ the new mapping $U_{0}: B^{+} \rightarrow \mathcal{M}$ given by

$$
U_{0}(z):=\left(u_{0}(z), \bar{z}+\tilde{\Phi}(z)\right)
$$

will then be conformally parametrized (w.r.t. the Riemannian metric on $\mathcal{M}$ ). 
The new functional $\tilde{I}$ will again be conformally invariant, and its coefficients are given by

$$
\tilde{G}_{j k}= \begin{cases}G_{j k} & , \quad 1 \leq j, k \leq N \\ \delta_{j k} & , \quad N+1 \leq j, k \leq N+2 \\ 0 & , \quad \text { otherwise }\end{cases}
$$

and

$$
\tilde{B}_{j k}= \begin{cases}B_{j k}, & 1 \leq j, k \leq N \\ 0, & \text { otherwise }\end{cases}
$$

Hence, for $U=(u, v)$ we have the decomposition

$$
\tilde{I}[U]=I[u]+D(v)
$$

and it is straightforward to check that the analogue of the Lemma in section 2 holds, i.e. $U_{0}$ is a stationary point of $\tilde{I}$.

To be able to apply the arguments from [11] one has to impose an additional compatibility condition on the supporting surface $S$ and the coefficients in the original functional $I$. In the case of the integral (3) one had to assume that the vector field $Q$ is tangential along $S$. This condition turns out to be equivalent to the fact that the surface $u_{0}\left(B^{+}\right)$intersects the supporting surface $S$ orthogonally along the free boundary $I$. However, since it is not clear that such a condition is really necessary we shall not be more explicit here.

Let us now turn our attention to the case of harmonic maps which are stationary for a free boundary problem in a Riemannian manifold. The corresponding problem for minimal surfaces (= conformally parametrized harmonic maps) has been investigated by J. Jost in [14] and [15]. First, we are going to consider the situation treated in [14]. There, Jost showed regularity for minimal surfaces in Riemannian manifolds of bounded geometry ${ }^{3}$ with a free boundary on a supporting hypersurface of class $C^{2}$ with bounded second fundamental form and a uniform neighbourhood in which the nearest point projection onto this hypersurface is uniquely defined. Obviously, this is the appropriate generalization of what we termed strict $C^{2}$-surface in $\mathbb{R}^{3}$ in section 2. In section 5 of [15] the result of [14] was generalized from the hypersurface situation to the case where the supporting surface is allowed to have arbitrary codimension $(\geq 1)$. Since

\footnotetext{
3 i.e. the sectional curvature is bounded and the injectivity radius is bounded away from zero. Vol. 15, $n^{\circ} 2-1998$.
} 
our method reduces the case of stationary harmonic maps to the case of stationary minimal surfaces in a higher dimensional manifold we may as well consider the situation where the supporting submanifold has arbitrary codimension. The reasoning illustrated in the model case will then give the following result which we state without proof.

Theorem 2. Suppose that $M$ is a complete n-dimensional Riemannian manifold of bounded geometry, $\Sigma$ is a $C^{2}$-submanifold of $M$ without boundary with bounded second fundamental form and a uniform neighbourhood in which the nearest point projection onto $\Sigma$ is uniquely defined. Let $\mathcal{C}(\Sigma):=\left\{X \in H_{2}^{1}\left(B^{+}, M\right): X(I) \subset \Sigma\right\}$ and assume that $X \in \mathcal{C}(\Sigma)$ is a stationary harmonic map in the sense of Definition 2. Then $X$ is of class $C^{1, \alpha}\left(B^{+} \cup I, M\right)$ for any $\alpha \in(0,1)$. If $\Sigma$ is of class $C^{3}$ then $X \in C^{2, \alpha}\left(B^{+} \cup I, M\right)$ for any $\alpha \in(0,1)$.

Remarks. -1 . For the proof one only has to note that as in section 2

$$
\Phi(w):=\left\langle X_{u}(w), X_{u}(w)\right\rangle-\left\langle X_{v}(w), X_{v}(w)\right\rangle-2 i\left(X_{u}(w), X_{v}(w)\right\rangle
$$

is again holomorphic on $B^{+}$, continuous in $B^{+} \cup I$, and real-valued on $I$. Here, $\langle\cdot, \cdot\rangle$ denotes the scalar product in the tangent space $T M$. Thus, we replace $M$ by $\tilde{M}:=M \times \mathbb{C}, \Sigma$ by $\tilde{\Sigma}:=\Sigma \times \mathbb{R} \subset \tilde{M}$, and $X$ by $Y$ where $Y(w):=(X(w), \bar{w}+\Phi(w))$; c.f. section 2 for the notation. Then we are locally in the situation considered in [14] respectively in section 5 of [15]. The result proved by Jost now implies Theorem 2 .

2. Our method also works in the piecewise smooth case considered in [15] and yields continuity just as for stationary minimal surfaces.

Instead of entering the general framework let us finally explain the case of a supporting surface with non-empty boundary in the Euclidean situation.

Here, one considers a surface $S \subset \mathbb{R}^{3}$ of class (at least) $C^{2}$ such that the boundary $\partial S$ of the manifold $S$ is a regular one-dimensional submanifold of class $C^{2}$ such that the following assumption is satisfied (compare [10] and section 7.6 of [2]).

Definition 3. - A supporting surface $S$ is said to fulfil Assumption (B) if the following holds true:

There are numbers $0<\rho_{0}<1, K_{0} \geq 0$, and $K \geq 1$ such that we have:

For each point $x_{0} \in S$ there is a (full) neighbourhood $\mathcal{U}$ of $x_{0}$ in $\mathbb{R}^{3}$ and a $C^{2}$-diffeomorphism $h$ of $\mathbb{R}^{3}$ onto itself with the properties (B1)-(B4).

(B1) The inverse $g:=h^{-1}$ maps $\mathcal{U}$ onto the open ball $B_{\rho_{0}}:=\left\{y \in \mathbb{R}^{3}\right.$ : $\left.|y|<\rho_{0}\right\}$ such that $g\left(x_{0}\right)=0$. 
(B2) There exists some number $\sigma=\sigma\left(x_{0}\right) \in[-1,0]$ such that

$$
g(S \cap \mathcal{U})=\left\{y \in B_{\rho_{0}}: y^{3}=0, y^{1} \geq \sigma\right\}
$$

and

$$
g(\partial S \cap \mathcal{U})=\left\{y \in B_{\rho_{0}}: y^{3}=0, y^{1}=\sigma\right\} .
$$

If $x_{0} \in \partial S$ then $\sigma=0$, and $\sigma \leq-\rho_{0}$ if $\partial S \cap \mathcal{U}=\varnothing$.

(B3) If $g_{j k}(y):=h_{y^{j}}(y) \cdot h_{y^{k}}(y)$ are the components of the fundamental tensor of $\mathbb{R}^{3}$ w.r.t. to the curvilinear coordinates $y$ then

$$
K^{-1}|\xi|^{2} \leq g_{j k}(y) \xi^{j} \xi^{k} \leq K|\xi|^{2}
$$

for all $\xi, y \in \mathbb{R}^{3}$.

(B4) For any $y \in \mathbb{R}^{3}$ and any $j, k, l \in\{1,2,3\}$ we have

$$
\left|\frac{\partial g_{j k}}{\partial y^{l}}\right|(y) \leq K_{0} \text {. }
$$

For the convenience of the reader we have chosen to give the precise definition as in [2], because it is now straightforward to check that the new supporting submanifold $\tilde{S}=S \times \mathbb{R} \subset \mathbb{R}^{5}=\mathbb{R}^{3} \times \mathbb{C}$ satisfies a similar condition. For example,

$$
\tilde{g}(\tilde{S} \cap \tilde{\mathcal{U}})=\left\{y \in B_{\rho_{0}}: y^{3}=y^{5}=0, y^{1} \geq \sigma\right\}
$$

and

$$
\tilde{g}(\partial \tilde{S} \cap \tilde{\mathcal{U}})=\left\{y \in B_{\rho_{0}}: y^{3}=y^{5}=0, y^{1}=\sigma\right\} .
$$

Here, $\tilde{\mathcal{U}}$ denotes a neighbourhood in $\mathbb{R}^{5}$ and $B_{\rho_{0}} \subset \mathbb{R}^{5}$ the open ball of radius $\rho_{0}$ centered at the origin. The class $\mathcal{C}(S)$ is defined exactly as in section 2. As before, if $Y: B^{+} \rightarrow \mathbb{R}^{5}$ is the new harmonic map the proof of $Y \in C^{0}\left(B^{+} \cup I, \mathbb{R}^{5}\right)$ proceeds in the same way as the proof of Theorem 1 in section 7.6 of [2] with the obvious slight modifications that are due to the fact that $\tilde{S} \subset \mathbb{R}^{5}$ is no longer a hypersurface.

To show Hölder-continuity of the original map $X$ one uses Widman's hole filling technique for the new map $Y$ which is conformally parametrized. Note, that the conformality condition now implies that

$$
\left|D Y^{1}\right|^{2} \leq K^{*}\left(\left|D Y^{2}\right|^{2}+\left|D Y^{3}\right|^{2}+\left|D Y^{4}\right|^{2}+\left|D Y^{5}\right|^{2}\right) .
$$

Using (12) the proof of Theorem 2 in section 7.6 of [2] then shows that $Y \in C^{0, \alpha}\left(B^{+} \cup I, \mathbb{R}^{5}\right)$ for some $\alpha \in(0,1)$, in particular the Hölder-continuity of $X$. 
THEOREM 3. - Let $S \subset \mathbb{R}^{3}$ be a supporting $C^{2}$-surface satisfying assumption (B), and suppose that $X \in \mathcal{C}(S)$ is a stationary harmonic map. Then, for some $\alpha \in(0,1)$ we have

$$
X \in C^{0, \alpha}\left(B^{+} \cup I, \mathbb{R}^{3}\right) .
$$

Remarks. - The question of higher regularity - note that $C^{1,1 / 2}$ is optimal in the minimal surface case if $\partial S \neq \varnothing$ - will not be discussed here. Instead, the interested reader is referred to section 7.7 of [2].

\section{REFERENCES}

[1] U. Dierkes, S. Hildebrandt, A. Küster and O. Wohlrab, Minimal Surfaces I. Grundlehren math. Wiss., Vol. 295, Springer-Verlag Berlin Heidelberg 1992.

[2] U. Dierkes, S. Hildebrandt, A. Küster and O. Wohlrab, Minimal Surfaces Il. Grundlehren math. Wiss., Vol. 296, Springer-Verlag Berlin Heidelberg 1992.

[3] G. Dzıuk, Über die Stetigkeit teilweise freier Minimalflächen. Manuscr. Math., Vol. 36, 1981, pp. 241-251.

[4] G. Dzıuk, $C^{2}$-Regularity for partially free minimal surfaces, Math. Z., Vol. 189, 1985, pp. $71-79$.

[5] M. Giaquinta and S. Hildebrandt, Calculus of Variations. Vol.1 , The Lagrangian formalism. Grundlehren math. Wiss., vol. 310 , Springer-Verlag Berlin Heidelberg 1995.

[6] M. GrütER, Regularity of weak H-surfaces. I. Reine Angew. Math. 329, 1981, pp. 1-15.

[7] M. GRörtek, Conformally invariant variational integrals and the removability of isolated singularities, Manuscr., Math., Vol. 47, 1984, pp. 85-104.

[8] M. GRUTER, Eine Bemerkung zur Regularität stationärer Punkte von konform invarianten Variationsintegralen, Manuscr. Math., Vol. 55, 1986, pp. 451-453.

[9] M. GRÜTER, The monotonicity formula in geometric measure theory, and an application to partially free boundary problems. In: S. Hildebrandt, R. Leis (editors), Partial differential equations and calculus of variations. Lect. Notes Math., Vol. 1357, Springer-Verlag Berlin Heidelberg 1988, pp. 238-255.

[10] M. Grüter, S. Hildebrandt and J. C. C. Nitsche, On the boundary behavior of minimal surfaces with a free boundary which are not minima of the area. Manuscr., Math., Vol. 35, 1981, pp. 387-410.

[11] M. Gruter, S. Hildebrandt and J. C. C. Nitsche, Regularity for surfaces of constant mean curvature with free boundaries. Acta Math., Vol. 156, 1986, pp. 119-152.

[12] F. HélEin, Régularité des applications faiblement harmoniques entre une surface et une variété riemannienne, C. R. Acad. Sci. Paris, Vol. 312, Série I, 1991, pp. 591-596.

[13] W. JÄGER, Behavior of minimal surfaces with free boundary, Commun, Pure Appl. Math., Vol. 23, 1970, pp. 803-818.

[14] J. JosT, On the regularity of minimal surfaces with free boundaries in a Ricmannian manifold, Manuscr. Math., Vol. 56, 1986, pp. 279-291.

[15] J. JosT, Continuity of minimal surfaces with piecewise smooth boundaries, Math. Ann.. Vol. 276, 1987, pp. 599-614.

[16] H. LEwY, On minimal surfaces with partially free boundary, Commun. Pure Appl. Math., Vol. 4, 1951, pp. 1-13.

[17] F. H. LiN, Une remarque sur l'application $x /|x|$, C. R. Acad. Sci. Paris, Vol. 305, Série I, 1987, pp. 529-531.

[18] C. B. MoRREY, Multiple integrals in the calculus of variations, Grundlehren math. Wiss., Vol. 130, Springer-Verlag Berlin Heidelberg 1966. 
[19] J. C. C. NitSCHE, Vorlesungen über Minimalflächen. Grundlehren math. Wiss., Vol. 199, Springer-Verlag Berlin Heidelberg 1975.

[20] J. C. C. NITSCHE, Lectures on minimal surfaces, vol. 1: Introduction, fundamentals, geometry and basic boundary problems. Cambridge Univ. Press 1989.

[21] J. QinG, Boundary regularity of weakly harmonic maps from surfaces, Journ. Funct. Anal., Vol. 114, 1993, pp. 458-466.

[22] R. SCHOEN, Analytic aspects of the harmonic map problem. In: S. S.Chern (editor), Seminar on nonlinear partial differential equations. Math. Sci. Res. Inst. Publ., Vol. 2. Springer-Verlag New York 1984, pp. 321-358.

[23] K. STEFFEN, Two-dimensional minimal surfaces and harmonic maps. In: G. Anzellotti, M. Giaquinta (editors), Lecture notes on geometric measure theory and geometrical variational problems. Lecture notes series, Vol. 4, Dipartimento di Matematica, Università degli Studi di Trento 1995, $40 \mathrm{pp}$.

(Manuscript received November 27, 1995.) 